\title{
Motion of Liquid Droplets/Film in the Gas Channels of a SO2 Module
}

\author{
Radu Cimpeanu ${ }^{1}$, Yousef Daneshbod ${ }^{2}$, Qingxia Li $^{3}$, Pejman Sanaei $^{4}$, Pavel \\ Dubovski $^{5}$, Ali Nadim ${ }^{6}$ and Marina Chugunova ${ }^{6} \dagger$ \\ 1 University of Warwick, UK \\ ${ }^{2}$ University of La Verne, California, USA \\ ${ }^{3}$ University of Alberta, Canada \\ ${ }^{4}$ New York Institute of Technology, New York, USA \\ ${ }^{5}$ Stevens Institute of Technology, New Jersey, USA \\ ${ }^{6}$ Claremont Graduate University, California, USA
}

(Communicated to MIIR on 26 August 2021)

Study Group: MPI 2018, 25-29 June 2018, Claremont, CA, USA

Communicated by: Hilary Ockendon

Industrial Partner: W.L. Gore \& Associates

Team Members: Radu Cimpeanu, University of Warwick; Marina Chugunova, Claremont Graduate University; Yousef Daneshbod, La Verne University; Qingxia Li, University of Alberta; Pejman Sanaei, New York Institute of Technology; Pavel Dubovski, Stevens Institute of technology Ali Nadim, Claremont Graduate University.

Industrial Sector: Materials processing

Key Words: Droplets, Contact angle, Coalescence

MSC2020 Codes: 76

\section{Summary}

Understanding of liquid droplets dynamics in gas channels is critical for improvement of performance and durability of the catalysts made of a dense porous material. This report describes a mathematical model for studying how different surface properties and operating conditions affect the dynamics of liquid droplets. We present multiple numerical simulations of a single droplet dynamics for different sizes of droplets and different choices of contact angles. We also study influence of an air flow to a thin liquid film and obtained travelling wave type solutions.

$\dagger$ Corresponding Author: Marina.Chugunova@cgu.edu 


\section{Final Report: Motion of Liquid Droplets/Film in the Gas Channels of a $\mathrm{SO}_{2}$ Module}

Prepared by Radu Cimpeanu, Yousef Daneshbod, Qingxia Li, Pejman Sanaei, Pavel Dubovski, Ali Nadim, Marina Chugunova

\section{Problem Description}

Catalysts are are an integral part of many chemical processes. Catalysts are usually made of a dense but porous material such as activated carbon, zeolites, etc. that provide a large surface area. Liquids that are produced as a byproduct of a gas reaction at the catalyst site transport to the surface of the porous material, slowing down transport of the gaseous reactants to the catalyst active site. Understanding the dynamics of the liquid droplet in the gas channel is critical to maintain performance and durability of the catalyst assembly.

Our goal in this workshop is to develop a mathematical model to understand how different surface properties and operating conditions affect the dynamics of the droplet or the formation of a liquid film. Specifically, we are interested in the physical conditions where the droplet moves up or down and where liquid films are formed.

\section{Modeling a Static Droplet}

We will begin by determining the shape of a static droplet hanging from a vertical wall in the presence of gravity. We assume that physical variations in the transverse $z$ direction are negligible, thereby a two dimensional model is used to describe the shape of the droplet (see Figure 1).

\subsection{Formulation}

A droplet at rest has no inertial or frictional stresses, therefore the Navier-Stokes equation assumes the simple form:

$$
\nabla P=\rho \boldsymbol{g} .
$$

Resolving equation (1) in the $x$ direction gives us

$$
\frac{\partial P}{\partial x}=0
$$

which implies,

$$
P=P(y)
$$

Using the fact that pressure is only a function of $y$, resolving equation (1) in the $y$ direction will lead to,

$$
\frac{d P}{d y}=-\rho g
$$

A simple integration of (4) will yield,

$$
P(y)=-\rho g y+\text { constant }
$$




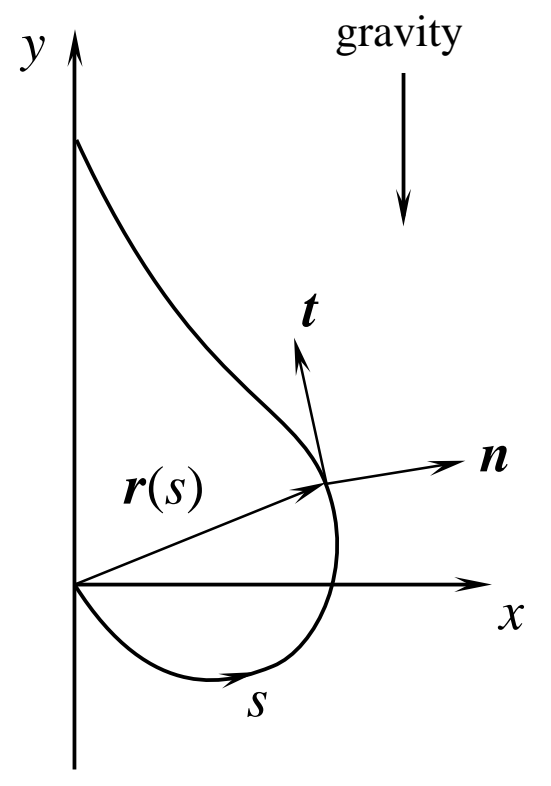

Figure 1: Schematic of a droplet hanging from a vertical wall. The origin of the $x y$ coordinate system is located on the bottom contact point of the droplet

Equation (5) is rather surprising because it tells us that the pressure inside a static droplet is a linear function of the altitude, independent of surface tension.

Surface tension acts on the surface of the droplet, consequently, it does not appear in the NavierStokes equations but rather enters through the boundary conditions. In the absence of gravity, this boundary condition is (the well-known Young-Laplace equation) expressed as:

$$
P(s)=P_{a}+\sigma \kappa(s),
$$

where $P(s)$ is the fluid pressure along the fluid-air interface, $P_{a}$ the atmospheric pressure outside the droplet, $\sigma$ the surface tension and $\kappa$ the curvature of the droplet which is a function of the arc length $s$. The position vector describing the geometry of the droplet can be expressed as,

$$
\boldsymbol{r}(s)=x(s) \boldsymbol{i}+y(s) \boldsymbol{j}
$$

where $i$ and $j$ are the unit vectors in the $x$ and $y$ directions respectively. The unit tangent $t$ can now be found by calculating the arc-rate change of the position vector. That is

$$
\boldsymbol{t}=\frac{d \boldsymbol{r}}{d s}=x^{\prime} \boldsymbol{i}+y^{\prime} \boldsymbol{j}
$$

Note that we have used the accent mark to denote derivative with respect to arc length; that is, in general $(\quad)^{\prime}=\frac{d}{d s}(\quad)$. The unit normal vector $\boldsymbol{n}$ can also be found as follows

$$
\boldsymbol{n}=\boldsymbol{t} \times \boldsymbol{k}=y^{\prime} \boldsymbol{i}-x^{\prime} \boldsymbol{j},
$$

where $\boldsymbol{k}$ is the unit vector in the $z$ direction. In addition, we can evaluate the curvature using any of the two following relationships

$$
\boldsymbol{t}^{\prime}=-\kappa \boldsymbol{n} \quad \text { or } \quad \boldsymbol{n}^{\prime}=\kappa \boldsymbol{t}
$$


The first relationship will give us

$$
-\kappa \boldsymbol{n}=x^{\prime \prime} \boldsymbol{i}+y^{\prime \prime} \boldsymbol{j} .
$$

Dot multiplying both sides of (11) by $\boldsymbol{n}$ will result in an equation for the curvature expressed as

$$
\kappa=x^{\prime} y^{\prime \prime}-x^{\prime \prime} y^{\prime}
$$

Upon substituting this into (1) we get

$$
\begin{gathered}
P-\rho g y=P_{a}+\sigma\left(x^{\prime} y^{\prime \prime}-x^{\prime \prime} y^{\prime}\right) \\
x^{\prime} y^{\prime \prime}-y^{\prime} x^{\prime \prime}+\frac{\rho g y}{\sigma} y=\frac{\Delta P}{\sigma}
\end{gathered}
$$

We define $l=\sqrt{\frac{\sigma}{\rho g}}$ as the capillary length.

$$
x^{\prime} y^{\prime \prime}-y^{\prime} x^{\prime \prime}+\frac{y}{l^{2}}=\frac{\Delta P}{\sigma}
$$

The equation can be written in a non-dimensional form by defining

$$
\tilde{x}=x / l, \quad \tilde{y}=y / l, \quad \tilde{s}=s / l \quad \text { and } \quad \Pi=l \Delta P / \sigma .
$$

Substituting the non-dimensional expressions (16) into equation (15) and removing the tilde symbols, we will have:

$$
x^{\prime} y^{\prime \prime}-y^{\prime} x^{\prime \prime}+y=\Pi \text {. }
$$

\subsection{Formulating the System of ODE's}

The second order ODE (17) involving two dependent variables $x$ and $y$ can now be transformed into a system of four first order ODE's by introducing the new dependent variables $u$ and $v$ as follows

$$
\begin{array}{cc}
x^{\prime}= & u \\
y^{\prime}= & v \\
u^{\prime}= & -v(\Pi-y) \\
v^{\prime}= & u(\Pi-y)
\end{array}
$$

along with the initial conditions,

$$
\begin{array}{lc}
x(0)= & 0 \\
y(0)= & 0 \\
u(0)= & \sin \theta \\
v(0)= & \cos \theta .
\end{array}
$$

where $\theta$ is the bottom contact angle of the droplet.

Note that in formulating the system of ODE's $(18)$, we have used the fact that $u^{2}+v^{2}=1$ or $u u^{\prime}+v v^{\prime}=0$.

\subsection{Shape Analysis for the Static Drop}

Below is the numerical result that we obtained for various contact angles. 


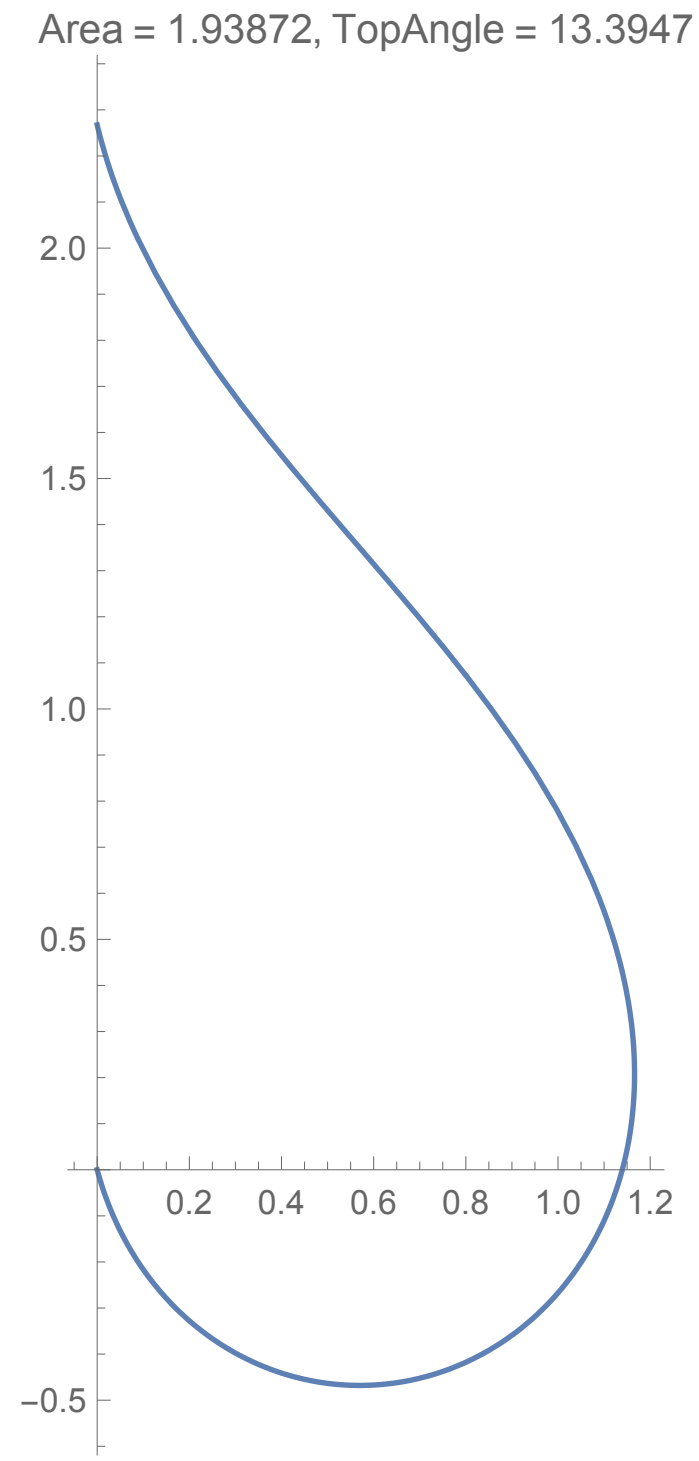

Figure 2: Contact angles $\theta=165$ and $\theta=13$.

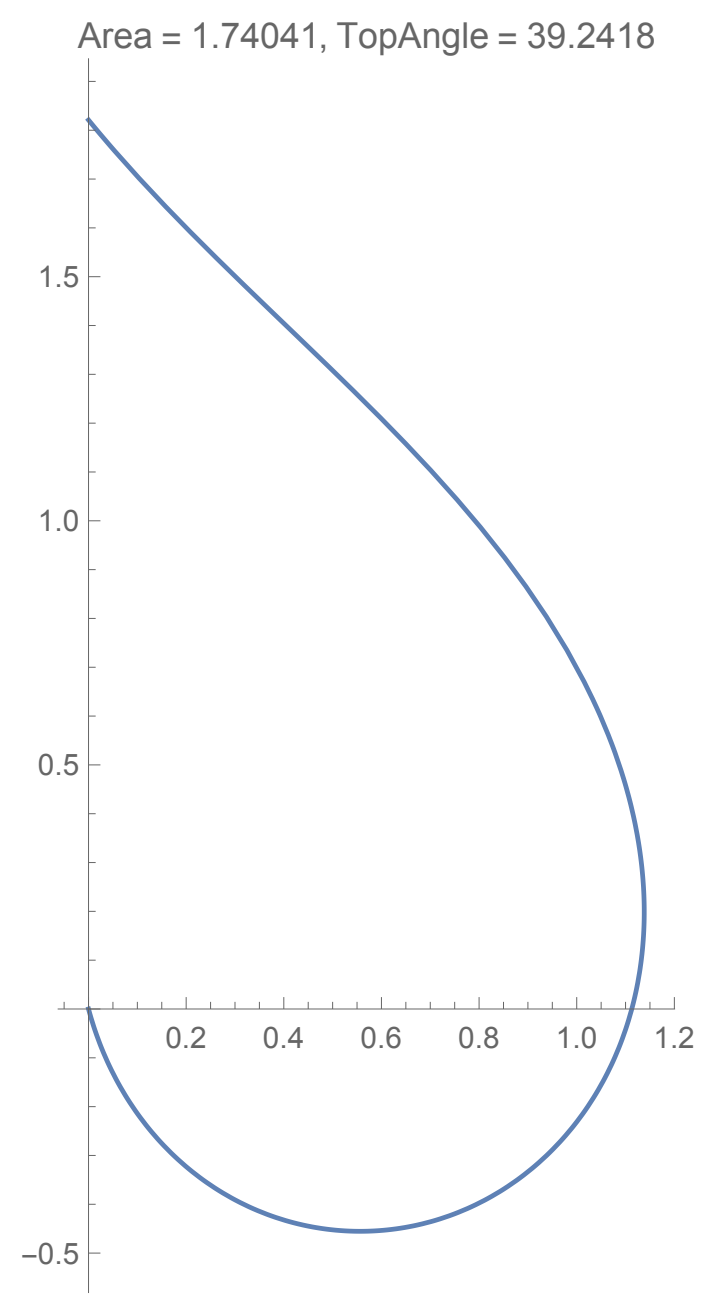

Figure 3: Contact angles $\theta=165$ and $\theta=39$. 


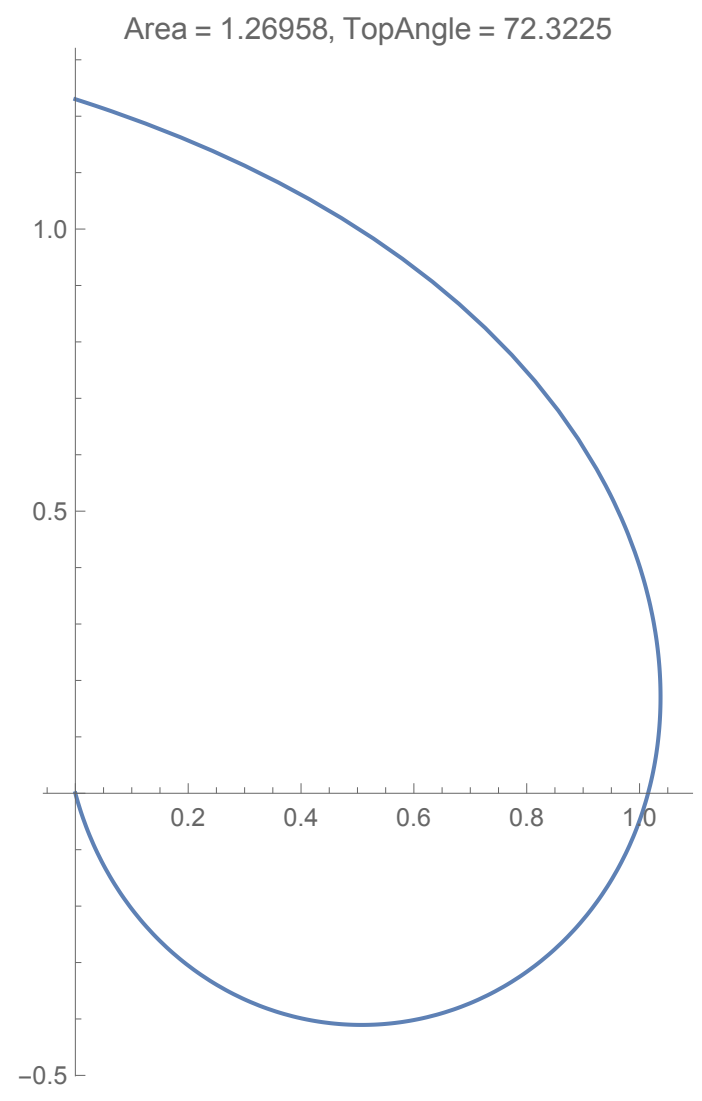

Figure 4: Contact angles $\theta=165$ and $\theta=72$.

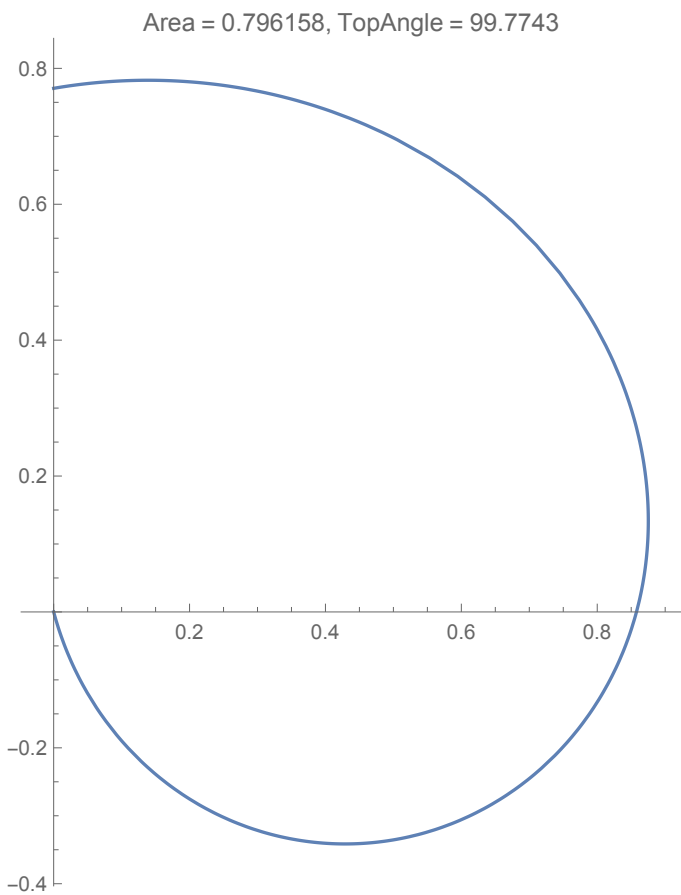

Figure 5: Contact angles $\theta=165$ and $\theta=100$. 


\section{Computational framework}

\subsection{Introduction}

From a general scientific standpoint, experimental, analytical, and numerical approaches are all instrumental in providing insight into aspects involving drop formation, evolution and interaction.

Most typically experimental methods give a direct line of investigation into the target flow properties, but are often restricted by laboratory time, equipment, video technology limitations and the sheer size of the parameter space.

Analytical techniques such as those discussed in previous subsections provide significant fundamental understanding, producing very useful predictive formulae, which can then drive innovation further in the design pipeline. While (at least ideally) simple to employ once available, they are often restricted to specific flow regimes and their derivation relies on certain simplifying assumptions which may become impractical when considering the real-life situation. Modelled processes in interfacial flows are often built on either linear or weakly nonlinear evolution hypotheses, ensuring the tractability of the mathematical formulation. Further progress can be made when a strong disparity in length- or timescales naturally occurs in the target systems (for example for long and thin droplets).

So-called "direct numerical simulations" provide a bridge between the two previously mentioned lines of investigation. They are "direct" because the full Navier-Stokes equations are solved, taking into account both gas and liquid properties, as well as the full set of interfacial conditions. In the early stages, numerical results can cross-validate analytical progress. In the late stages, accurate numerical experiments delve into nonlinear regimes beyond the reach of other techniques, offering detailed information at any timestep and from angles difficult to construct experimentally. Although they need significant computational resources (processing power, memory, data handling algorithms), they serve to reduce the search space, guiding experimental investigations in the right direction.

The present section provides an overview of the numerical methodology used in this study in order to drive our analytical and mathematical modelling efforts towards the highly nonlinear and complex regimes in the device presented as part of this workshop.

\subsection{Direct numerical simulations}

The volume-of-fluid (VOF) method was introduced by DeBar(1974) and has proved to be one of the historical advances in the computational study of interfacial flows. In this section we concisely present relevant concepts of the technique in the context of our investigation. We also introduce technical aspects behind the implementation of the method in Gerris and highlight prominent numerical and in general, algorithmical, features of the package.

The Gerris Flow Solver is an open-source stand-alone software package with tremendous resources in terms of both numerical modelling and computational performance, with an architecture oriented towards solving fluid flow problems. The source code is based on the $\mathrm{C}$ language and is freely available under the Free Software GPL license. It was created by Popinet (2003), with the support of the National Institute of Water and Atmospheric research (NIWA) in New Zealand and Institut Jean le Rond d'Alembert in Paris.

At this point we introduce the mathematical formulation as implemented in Gerris. We briefly sketch the approach for our particular problem in the present section and refer the reader to Popinet 
(2009) for details. The equations of motion are

$$
\begin{aligned}
\tilde{\rho}\left(\tilde{\mathbf{u}}_{t}+\tilde{\mathbf{u}} \cdot \nabla \tilde{\mathbf{u}}\right) & =-\nabla \tilde{p}+\nabla \cdot(2 \tilde{\mu} \mathcal{D})+\tilde{\sigma} \kappa \delta_{s} \mathbf{n}+\mathbf{F}_{e} \\
\tilde{\rho}_{t}+\nabla \cdot(\tilde{\rho} \tilde{\mathbf{u}}) & =0 \\
\nabla \cdot \tilde{\mathbf{u}} & =0
\end{aligned}
$$

where $\mathcal{D}$ is the rate of strain tensor $\mathcal{D}_{i j}=(1 / 2)\left(\partial \tilde{u}_{i} / \partial x_{j}+\partial \tilde{u}_{j} / \partial x_{i}\right)$. All interfacial forces are transferred to the momentum equations in what is commonly called the "one-fluid" formulation - see Tryggvason et al. (2011). The physical properties describing each fluid (density, viscosity etc.) are included by singular distributions and the same set of equations (19) accounts for the entire domain. The Dirac distribution $\delta_{s}$ isolates the surface tension effects to the interface alone, and external forces (such as gravity) may be included via the $\mathbf{F}_{e}$ term.

In volume-of-fluid methods, relevant properties such as density or viscosity are represented in terms of a volume fraction $c(\mathbf{x}, t)$, where $c$ is a generic colour function which takes the value 0 in one fluid and 1 in the other. More specifically we write

$$
\begin{aligned}
\tilde{\rho}(c) & \equiv c \tilde{\rho}_{1}+(1-c) \tilde{\rho}_{2}, \\
\tilde{\mu}(c) & \equiv c \tilde{\mu}_{1}+(1-c) \tilde{\mu}_{2},
\end{aligned}
$$

Under this treatment, a density equation (the others properties are treated in a similar manner) of the general form

$$
\tilde{\rho}_{t}+\nabla \cdot(\tilde{\rho} \tilde{\mathbf{u}})=0
$$

becomes

$$
c_{t}+\nabla \cdot(c \tilde{\mathbf{u}})=0,
$$

which is solved for $c$ and the results substituted into (20)-(21). The value of $c$ is interpolated across the interface by introducing a small transition layer in its vicinity to smooth the variation of quantities from one region to the other. The sharp transitions in physical properties at the interface are relaxed numerically by applying a filter operator (smoothing) within a single grid cell, thus making grid refinement a crucial factor in obtaining accurate solutions. It is important to underline that the fluid flow equations are solved in both media (here the droplet and the gas flow) and the typical interfacial conditions considered at the interface itself are:

- continuity of velocities;

- kinematic condition;

- contiunity of normal and tangential stresses.

There are some multi-physics capabilities, such as for example the addition of electric fields (see López-Herrera et al. (2011) for details), which naturally add further complexity to the problem, including the presented set of conditions at the interface and their coupling to the flow problem itself.

Gerris is one of the few available open-source packages incorporating dynamic mesh adaptivity. Once a base mesh is created, the level of refinement is described using quadtrees in $2 \mathrm{D}$ or octrees in 3D. This tree structure provides not only a solid tracking possibility of how refinement occurs, but also allows for efficient parallel processing. The user can specify a maximum degree of refinement which will represent the depth of the graph. This very beneficial feature reduces the number of degrees of freedom by several orders of magnitude. 
The quadtree (octree in three dimensions) structure was adopted from the onset with the aim to provide a suitable numerical environment for massively parallel computations. The MPI (Message Parsing Interface) library is employed for parallelisation purposes and solid scalability is implemented using a load-balancing algorithm. For further information regarding the technical aspects of the parallelisation in Gerris we refer the reader to Agbaglah et al. (2011).

The Gerris (Popinet (2003), Popinet (2009)) and, more recently, Basilisk Flow Solvers are opensource freeware packages with tremendous resources in terms of both numerical modelling and computational performance. Their qualities, in particular in terms of adaptive mesh refinement (AMR), become evident in the study of the problem of drop dynamics in high speed flows. Capturing the evolution of the fluid-fluid interfaces demands an appropriate resolution, accounting for possible topological transitions. A highly refined uniform mesh spanning a large region of computational domain would make the problem intractable. Thus the combination between the volume-of-fluid technique and adaptive mesh refinement is perfectly suited for the problem at hand; in their absence highly accurate calculations at realistic parameter values would become unrealisable with present day computing architectures.

Finally, it is worth underlining that this is not what is commonly referred to as "black-box software", in which using a graphical interface and button-based interaction produces results with an often unknown underlying methodology. Instead, the work here is built on the basis of source code implementation in a transparent environment suitable for both fundamental scientific studies, as well as advances in practical directions. For example, the author has published mathematical work aimed at high-impact high-risk applications in a variety of areas ranging from electrohydrodynamic control of interfaces at small scales (Cimpeanu et al. (2014); Cimpeanu \& Papageorgiou (2015)) to high speed drop impact studies for the study of icing in the aircraft industry (Cimpeanu \& Papageorgiou (2018)).

\subsection{Geometry}

The computational domain aimed at replicating the gas channels of the filter is presented in Fig. 6 . Symmetry conditions on the top boundary are assumed in order to reduce the computational effort. On the bottom boundary, impermeability and no-slip conditions $(\mathbf{u}=\mathbf{0})$ are imposed. An inflow velocity profile is prescribed in the form of a laminar Poiseuille flow, with $3 \mathrm{~m} / \mathrm{s}$ taken to be the reference velocity at the centerline. Sufficiently downstream of this condition, a liquid droplet - typically of $\mathcal{O}(1) \mathrm{mm}$ in radius - is initialised, which is subjected to this oncoming gas flow, while at the same time being affected by gravity acting in the opposite direction. The balance between the droplet falling under gravity and the effect of the gas flow is one of the key elements of the problem. Further downstream an outflow condition (zero pressure and zero Neumann condition for the normal component of the velocity field) is added.

The anticipated mesh refinement around the liquid interface is complemented by adaptivity based on changes in velocity and vorticity, thus increasing the number of degrees of freedom in the calculation where the flow demands such detail. A typical two-dimensional run, spanning several tenths of a second in dimensional time, requires $\mathcal{O}\left(10^{4}\right)$ cells and was run for several hours on high performance computing clusters based in the UK.

An important technical aspect in this problem is the treatment of the contact angle dynamics. For simplicity, both advancing and receding contact angles of the drop are prescribed as constants (but varied from case to case). Having observed the static shapes modelled in the previous section, the capability of these having different values is also important and has been implemented and utilised, as 
Symmetry line

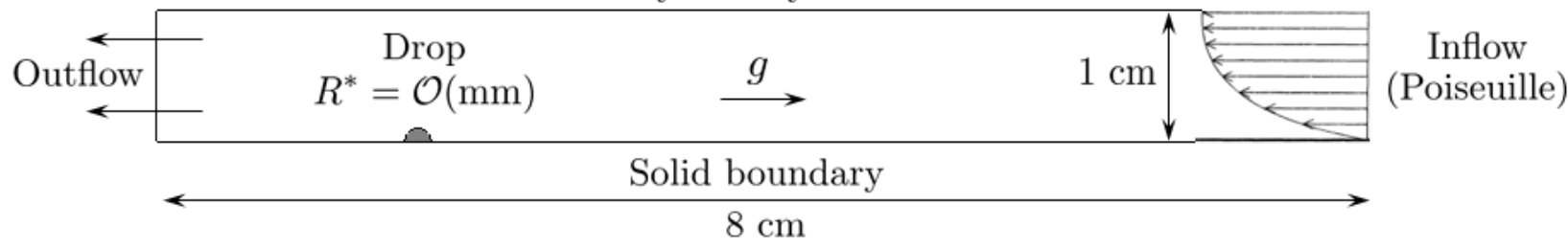

Figure 6: Schematic of the computational domain.

will be expanded on in the Results subsection. More sophisticated dynamic contact angle models can also be used (e.g. Afkhami et al. (2009)). With the angles fixed, numerical slip equal to the tangential velocity in the domain one half-cell distance away from the solid surface is then imposed, allowing the movement of the drop. The no-slip (or rather numerical slip) condition can be replaced by the classical Navier slip condition, with a prescribed slip length potentially modelling the non-trivial nature of the surface in a simplified manner.

In view of the long timescales of the droplet growth (hours to days in the experimental materials provided) as compared to the relatively short timescales simulated here (up to seconds) we have chosen to disregard changes in volume of the liquid. These can however be added by means of introducing blowing or suction conditions of the surface, should this feature become of interest or the growth rates be accelerated significantly due to different chemical reactions inside the pores.

\subsection{Results}

A first investigative goal is understanding the effect of the droplet size in the system. With all other parameters fixed (gas flow, contact angles at $\theta=90^{\circ}$ etc.), the droplet radius was varied from $1 / 8 \mathrm{~mm}$ to $4 \mathrm{~mm}$, interval suggested by previous experimental work. Intuitively we anticipate that:

- gravitational acceleration will become more significant as the drop size increases;

- the gas flow acting in the opposite direction will also play a more prominent role, as the drop itself lies in regions of stronger air movement;

- surface tension dictates the shape of the drop, with the smaller drops expected to remain approximately circular, while the larger drops should deform as inertial effects begin to dominate over capillarity.

In light of the above, a modified Froude number $F r^{*}=\rho_{\text {drop }} g R_{\text {drop }} /\left(\rho_{\text {gas }} V_{\text {gas }}^{2}\right)$ provides a useful dimensionless grouping comparing gravity acting on the drop to the inertial effect of the gas flow. The results of the parameter study are illustrated in Fig. 7 .

Several interesting features arise from this study. Before we begin the characterisation, it should be pointed out that the velocities in question are the extracted steady state velocities, after an initial transient of several tenths of a second have already passed and the liquid settles into a uniform motion. To begin with, small drops (below approximately $100 \mu \mathrm{m}$ in radius) do not move, as the competing effects are barely felt by the droplet. As the size of the drop increases and $F r^{*}=\mathcal{O}(1)$ the circular shape of the drop is still preserved, but its mass becomes significant. By contrast, the local gas velocity is still quite small (given its increase from approximately zero close to the surface) and as such the droplet slowly slides down the surface, with velocities of below $6 \%$ as compared to the reference $3 \mathrm{~m} / \mathrm{s}$ velocity in the 


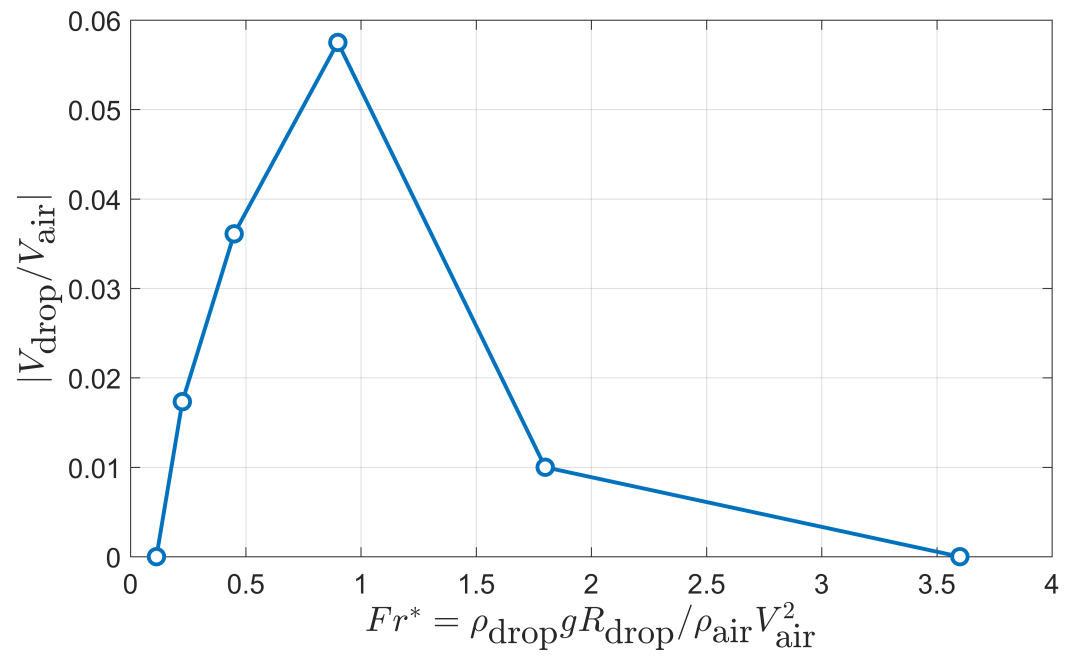

Figure 7: Relative steady state vertical velocity comparison between the center of mass of the drop (sliding down) and the surrounding gas flow (directed upwards) as a function of the key dimensionless grouping in the problem, the Froude number $F r^{*}$. With all other parameters fixed, this is essentially a study of the variation in droplet size, with radii increasing from $1 / 8 \mathrm{~mm}$ to $4 \mathrm{~mm}$ in factors of two.

system. Perhaps unexpectedly, a sudden decrease in the sliding velocity is then experienced. This is actually due to the drop now protruding more into the flow, which causes the advancing part of the drop to be pushed upwards, while the receding side is still falling. This translates to a progressively smaller contact radius and an elongation of the drop into the gas channel until ultimately the drop breaks into smaller liquid fragments which either adhere to the surface as smaller drops or are carried away by the gas flow.

While the above is a very useful indication of the size effect in the problem, an important restriction has been imposed in the form of the contact angles driving the drop motion. It would consequently be of interest to examine the flow sensitivity to this feature for the most delicate case in which $\mathrm{Fr}^{*} \approx 1$ and the balance of forces can easily be perturbed.

To this end, we consider the following batch of test cases, both inspired by previous theoretical insight and by the nature of the physical problem itself:

1. the standard neutral circular shape, with $\theta_{a}=\theta_{r}=90^{\circ}$;

2. the surface having a hydrophilic character, with the drop spreading according to $\theta_{a}=\theta_{r}=30^{\circ}$;

3. the surface having a hydrophobic character, with the drop decreasing its footprint according to $\theta_{a}=\theta_{r}=150^{\circ}$;

4. inheriting different receding and advancing contact angle values $\left(\theta_{a} \approx 110^{\circ}, \theta_{r}=98^{\circ}\right)$ for a drop of the appropriate size as given by the derived static drop model, as detailed in Fig. 8,

5. non-flat surface, with structural variations in a sinusoidal pattern (with a wavelength smaller than the drop radius). In this case the contact angle with the surface is fixed again at $90^{\circ}$, however the curved substrate introduces an additional complexity in how the drop would settle to this value. 


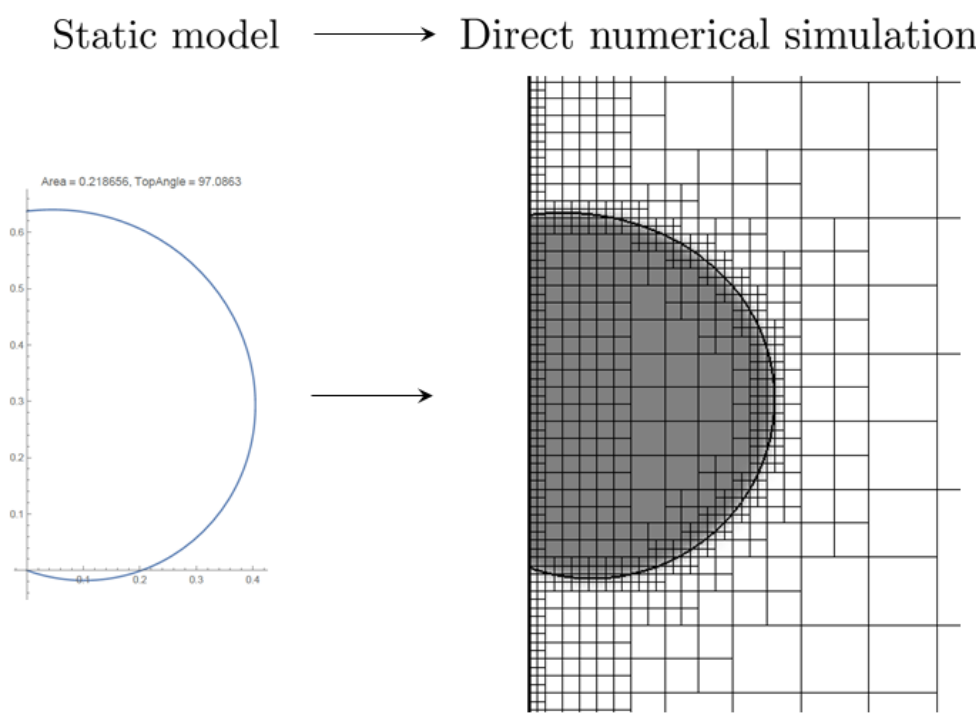

Figure 8: Sketch of the transfer of information (the advancing and receding contact angle values defining the droplet shape) from the model to the full two-dimensional problem as implemented as part of the direct numerical simulations.

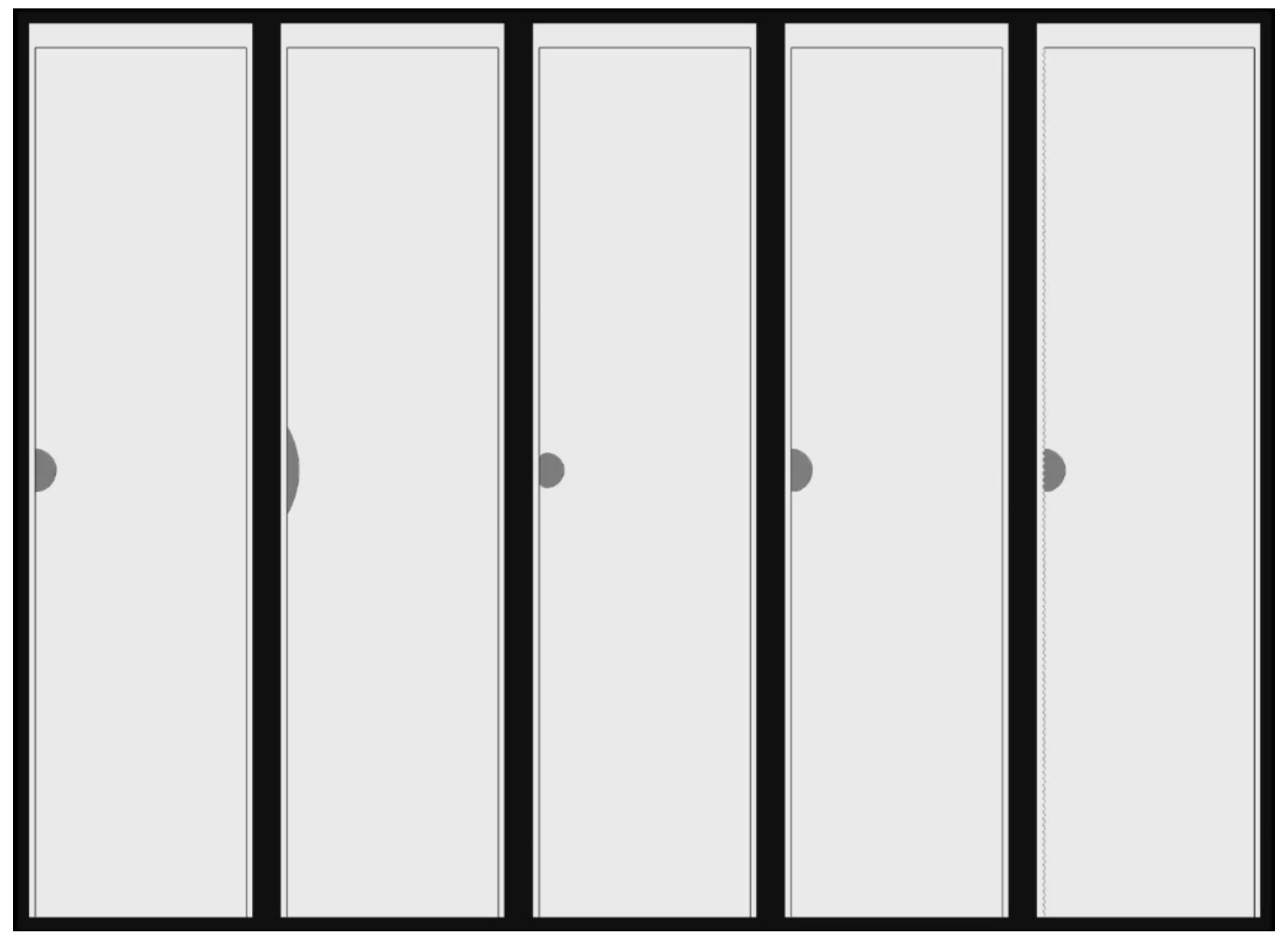

Figure 9: Summary of the test cases considered as part of the main single droplet study. From left to right: neutral contact angle $\left(\theta=90^{\circ}\right)$, hydrophilic drop $\left(\theta=30^{\circ}\right)$, hydrophobic drop $\left(\theta=150^{\circ}\right)$, inherited static drop angle values $\left(\theta_{a} \approx 110^{\circ}, \theta_{r}=98^{\circ}\right)$ and drop on a surface with a wavy/sinusoidal topography. 
In each case at initialisation the drop radius is set to $1 \mathrm{~mm}$, thus isolating the observations to a single parameter modification. A relevant snapshot of the early stage of the direct numerical simulations is shown in Fig. 9, while the supplementary video materials present the time evolution of these cases side by side.
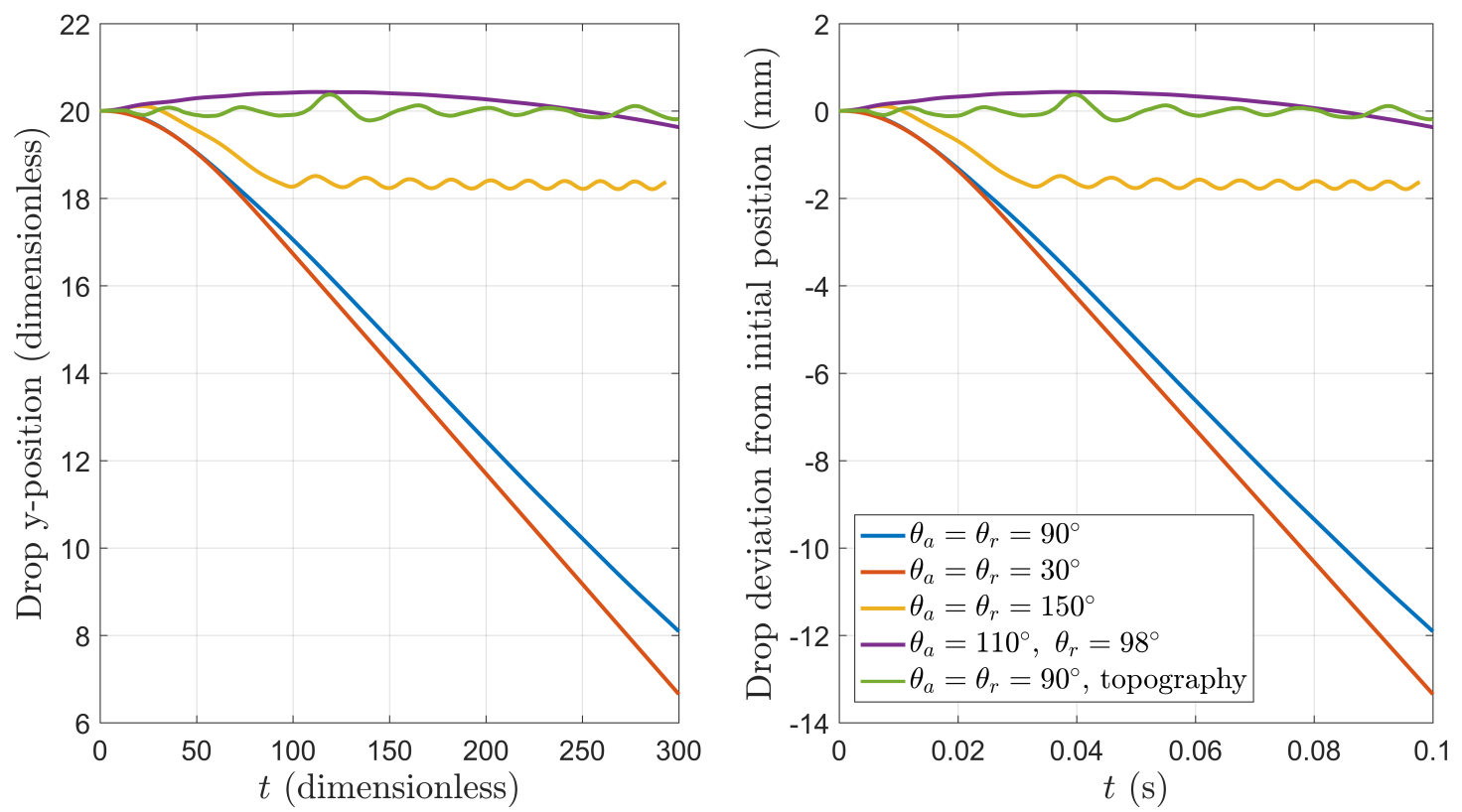

Figure 10: Evolution of the vertical coordinate of the center of mass for the drops defined in the five cases presented in Fig. 9

Quantitatively, the results are presented in Fig. 10,11, with the position of the center of mass (the vertical coordinate if gravity is pointing down and the gas flow is imposed upwards) and its velocity being calculated in each of the five cases. The raw data is shown on the left hand side of each figure (in dimensionless terms, with the drop radius used as reference lengthscale and $R_{\text {drop }} / V_{\text {gas }}$ used as reference timescale). One can also notice the fact that initially the vertical position of the drop is set to be 20 radii above the location of the inflow boundary. To improve interpretation however, the values have been re-dimensionalised and the coordinate system of the geometry repositioned at the initial center of mass of the drop in the right hand side subfigures in each case.

Starting from the case in which $\theta=90^{\circ}$ contact angles are imposed for both advancing and receding fronts, we are not surprised to find the more detailed perspective on the previous result, namely the drop sliding down the surface with an approximately constant velocity, found to have a magnitude of $0.12-0.13 \mathrm{~m} / \mathrm{s}$. The small variations owe themselves to the action of the external gas flow. It is interesting to note that in the case of the hydrophilic surface, the initial evolution over the first $0.02 \mathrm{~s}$ almost perfectly mirrors the previous setup. However in this case, because the drop is flatter, the effects of the gas are negligible and the drop slides under gravity at a very steady pace, described by a velocity of $0.15 \mathrm{~m} / \mathrm{s}$. A rather striking behaviour emerges from the other side of the spectrum in terms of contact angles, namely for the hydrophobic surface. With a relatively small contact area, the drop bulges and finds itself deeper into the gas flow. As it experiences stronger velocities, it proceeds to completely detach from the surface, moving into the gas flow in a periodic manner. Because of its mass, the drop will want to move in the direction of gravity as it is close to the surface and the gas velocity is small, 

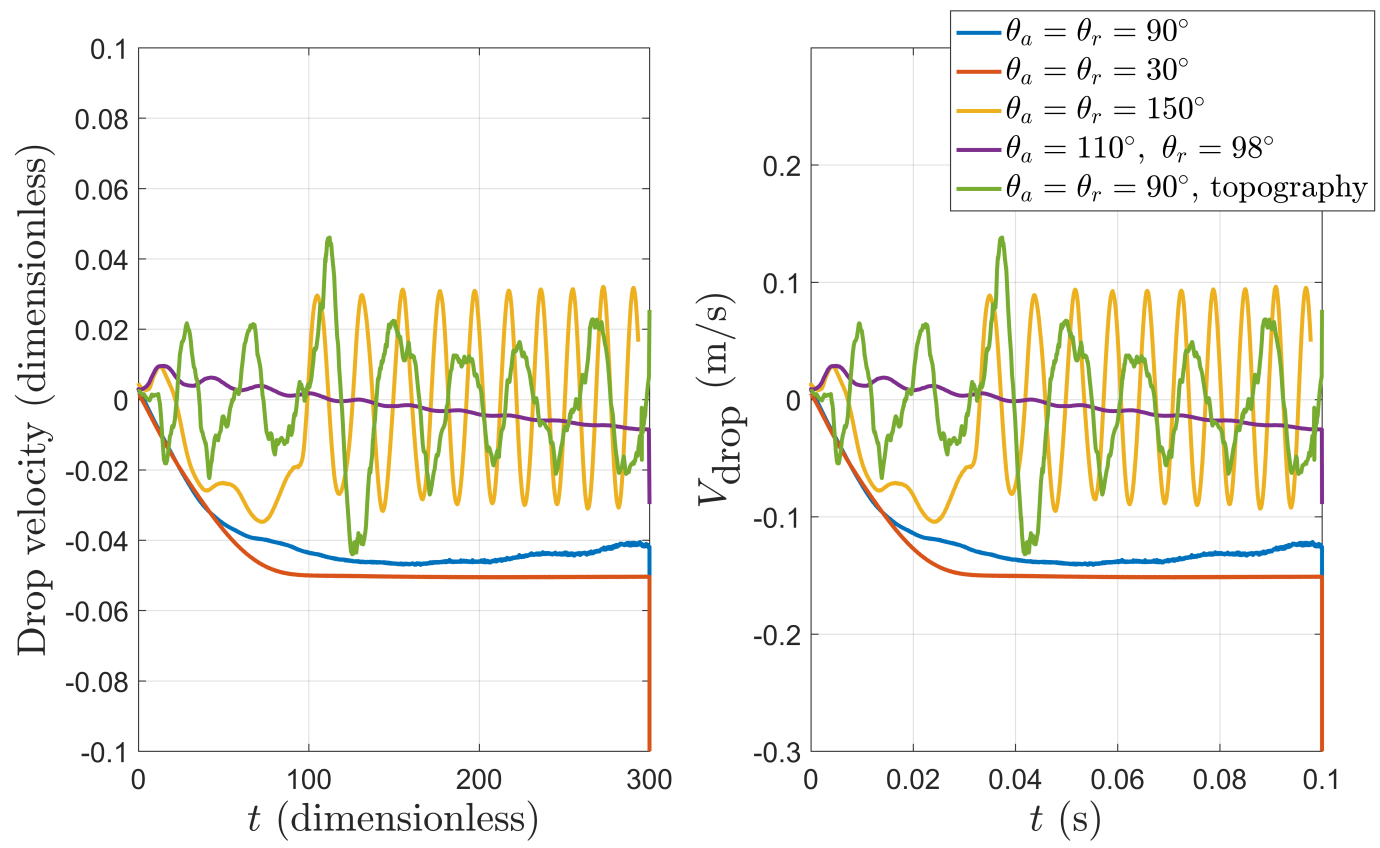

Figure 11: Evolution of the vertical velocity of the drops defined in the five cases presented in Fig. 9 .

however the gas attempting to squeeze between the liquid and the channel wall pushes it back into stronger background velocity regions and a repeated ellipsoidal trajectory is found for the center of mass, characterised by oscillatory movement of the vertical coordinate of the center of mass and its velocity. The drop is still small enough to maintain a roughly circular shape during this evolution. This is an interesting fundamental finding in its own right and perhaps an unexpected regime in the system, in which equilibrium solutions away from the surface are found that neither fall under gravity nor are expelled by the strong background flow, but rather develop over the fine local balance between the two effects.

Another - perhaps more predictable - type of behaviour found in the system is given by the case when the imposed contact angles are inherited from the static drop model. Recall that this is the solution that should emerge over long timescales by incorporating some of the key forces involved, such as gravity and surface tension, however in the absence of inertial effects or the background gas flow. While an initial adjustment period is still required, we find a comparatively small variation in the position of the drop, with the two contact regions successfully counterbalancing one another in terms of force directionality and the added affects providing only minor modifications to this equilibrium. This is both a useful validation point and a strong endorsement of the hybrid approach, in which the fast model can be used to detect the appropriate equilibrium contact angles, which are then inherited by the full simulation, with further effects dictating small refinements to the initially imposed values. Otherwise raw imposition of specific values in the direct numerical simulation platform would be both time-consuming, and, as observed, may artificially steer the observed dynamics in a certain direction. Such a tool is best utilised when in combination with model and experimental information.

We have also considered a change in the geometrical properties of the surface as opposed to chemical or fluid dynamical effects leading to changes in contact angle. In particular, due to the underlying pore structure, a flat surface may not be reflective of the realistic substrate below the drop. In a simplified setting, this has been included as a regular sinusoidal perturbation, on top of which the drop is 
allowed to freely find its equilibrium position. Naturally, both the wavelength and the amplitude of the imposed surface variation will play a significant role in the ensuing dynamics, further adding to the list of parameters in the problem. When present, such surface features were found to completely dominate the drop motion, with the contact line migrating between neighbouring local minima in a relatively ordered periodic manner, with the previously studied effects becoming secondary and the drop surface oscillating strongly as a result of this motion, but not deviating considerably from its initial position in terms of center of mass.

The studied system has revealed a fascinating zoo of behaviours, many of which could become the subject of much more careful scrutiny and potentially leading to interesting new discoveries from both mathematical and engineering perspectives.

\subsection{Conclusions and future work}

The implementation developed during the workshop has been used successfully both as an exploratory tool and in conjunction with the static drop model to synergistically consolidate our understanding of the flow. We have improved our knowledge of the single drop dynamics and the role of contact angles, with these parameters proving to be one of the key issues of the problem. Furthermore, we found the interaction with the non-trivial surface itself a worthwhile research direction.

A number of promising follow-up directions may be considered at this stage. From the perspective of the single drop analysis:

- use the model components and numerical results to improve the drop shape prediction;

- inspect the key flow variables to improve our understanding of the force balances and identify new regimes;

- model a suitable surface topography that would better emulate the modelled system;

- conduct parameter studies in appropriate ranges to classify the drop movement in the operational envelope of interest.

Initial attempts of modelling the interaction of multiple drops have also been designed. In this case some of the most interesting questions already to arise are:

- characterising the changes in the gas flow experienced by a given drop in the presence of additional liquid volumes further upstream;

- coalescence events between droplets and changes in behaviour as a result of the added volume.

Finally, two-dimensional modelling and computations underlie much of the progress above. There are exciting ramifications (and difficulties) when moving into three dimensions. From a mathematical standpoint, aspects related to the contact angle dynamics become considerably more involved, while numerically the significant increase in the number of degrees of freedom result in a much more challenging development, testing and investigative environment. Extensions to the existing capabilities, as well as modelling and experimental insight would however help guide such progress in the future, which may hold strong potential from both fundamental and practical perspectives. 


\section{Modeling the Formation of a Thin Film}

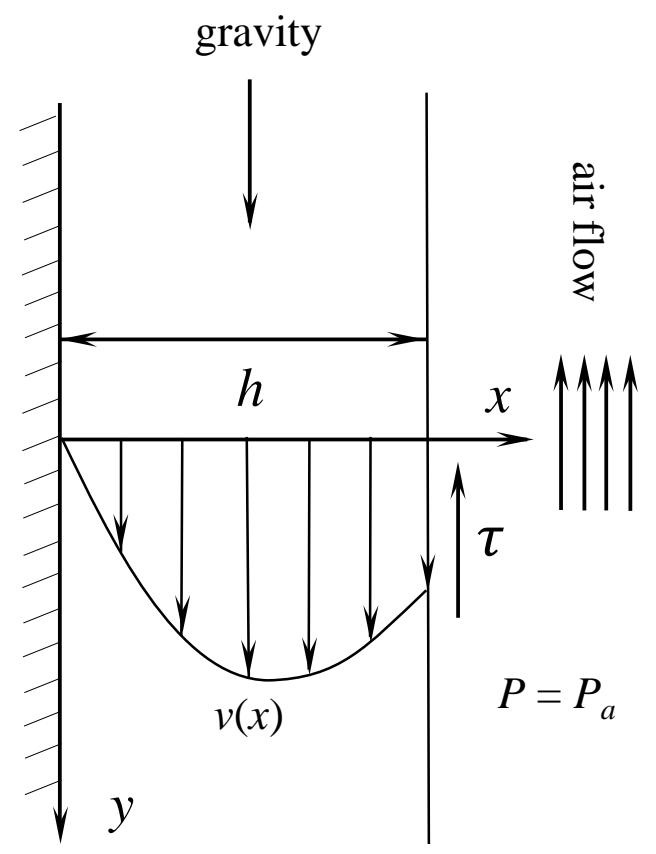

Figure 12: Velocity profile in film $v(x)$

In modeling the formation of the thin film we will use the so-called lubrication theory approximation. This approximation is often used when one length scale is significantly smaller than the other.

For example, if $h$ is the characteristic thickness of the film and $L$ the characteristic substrate length, then the key requirement for the lubrication theory to work is that the ratio $\epsilon=h / L$ be small (that is $\epsilon \ll 1$ ). When the Navier-Stokes equations are expanded in terms of this small parameter $\epsilon$, we will have

$$
\mu \frac{d^{2} v}{d x^{2}}+\rho g=0
$$

and

$$
\frac{\partial P}{\partial x}=0
$$

where $\mu$ and $\rho$ are the viscosity and density of the liquid respectively and $v=v(x)$ is the vertical velocity which is assumed positive facing downwards (see Figure 12). Using the prime notation for derivatives with respect to $x$, equation 24 can be written as

$$
v^{\prime \prime}=-\frac{\rho g}{\mu} .
$$

Integrating (26) twice will result in

$$
v(x)=-\frac{\rho g}{2 \mu} x^{2}+C x+D
$$


The no-slip condition at $x=0$ implies that $D=0$. The shear stress (drag) $\tau$ caused by the upward airflow at the interface is expressed as

$$
\tau=-\left.\mu \frac{d v}{d x}\right|_{x=h} .
$$

Therefore the constant $C$ can be found in terms of $\tau$ as follows:

$$
\tau=-\mu\left(-\frac{\rho g}{\mu}+C\right)
$$

which will eventually result in

$$
C=-\frac{\tau}{\mu}+\frac{\rho g h}{\mu}
$$

Then the velocity function $v(x)$ can be written as:

$$
\begin{aligned}
v(x) & =-\frac{\rho g}{2 \mu} x^{2}+\frac{\rho g h}{\mu} x-\frac{\tau}{\mu} x \\
& =-\frac{\rho g}{2 \mu}\left(x^{2}-2 h x\right)-\frac{\tau}{\mu} x
\end{aligned}
$$

Now if we calculate a flux per unit depth

$$
\begin{aligned}
q & =\int_{0}^{h} v(x) d x \\
& =-\frac{\rho g}{2 \mu}\left(\frac{h^{3}}{3}-h^{3}\right)-\frac{\tau}{\mu} \frac{h^{2}}{2} \\
& =\frac{\rho g}{2 \mu} h^{3}-\frac{1}{2} \frac{\tau}{\mu} h^{2}
\end{aligned}
$$

For a steady state $h=h^{*}$, we will have $q=0, h^{*}=\frac{3 \tau}{2 \rho g}$. If $\tau=0$, then profile will look like

$$
q(h)=\frac{\rho g}{3 \mu} h^{3}-\frac{1}{2} \frac{\tau}{\mu} h^{2}
$$

slowly varying $h, h=h(y, t), q(h(y, t))$ flux

$$
\text { ******************** }
$$

\section{Change coordinates:}

Let $x$ is parallel to wall. Then we will tackle the physical meaning of $\tau$.

Shear stress $\tau$ is defined as:

$$
\begin{aligned}
\tau & =\left.\mu_{\text {air }} \frac{\partial v_{\text {air }}}{\partial x}\right|_{\text {wall }} \\
& =\frac{\mu_{\text {air }} v_{\text {air }}}{H}
\end{aligned}
$$

for parabolic flow which can be computed analytically.

Question: Is there a steady solution?

$$
\begin{aligned}
\frac{\partial h}{\partial t} & =0 \\
\frac{\partial}{\partial x}\left(\alpha h^{3}-\beta h^{2}\right) & =S \\
\alpha h^{3}-\beta h^{2} & =S x+C
\end{aligned}
$$

If $h=h^{*}=\frac{\beta}{\alpha}$ at the top where $x=0$, then it is obvious to calculate that $C=0$. 


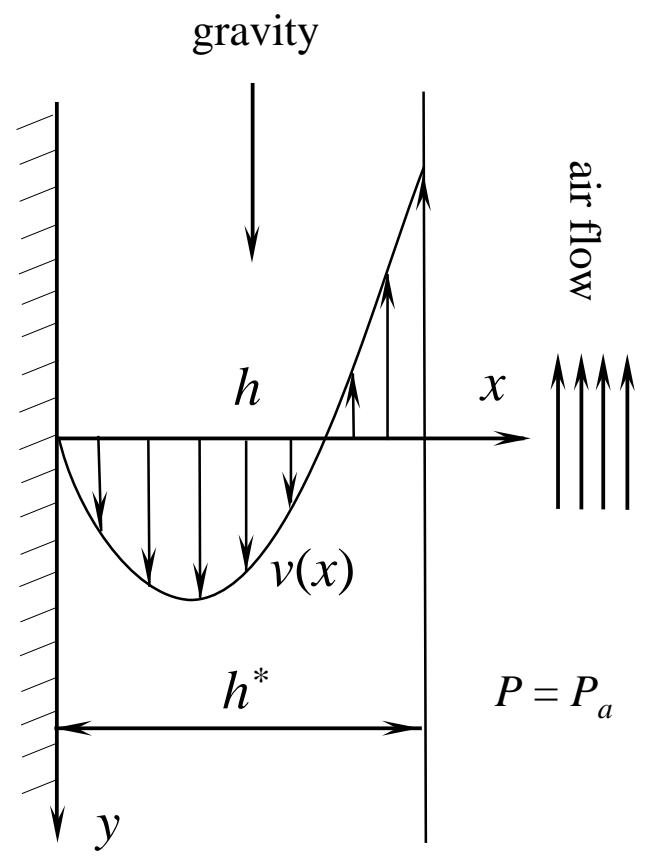

Figure 13: Velocity profile $v(x)$ in in which the flow rate $q=0$

\subsection{Thin film models with and without surface tension}

If the surface tension effect is neglected than $h(x, t)$ height of the thin liquid film can be modeled by:

$$
\frac{\partial h}{\partial t}+\left(3 h^{2}-2 h\right) \frac{\partial h}{\partial x}=S(x)
$$

where the convection term describes the competition between the air-flow blowing up the wall and the gravitational force that is pooling the film down. If the source term is weak $S(x)=S \leq 4 / 27$ and compactly supported on $[0,1]$ one can expect that the wall will stay completely dry below 0 and all the liquid will be pushed up the wall by the air flow, on the other side if the source term is strong $S(x)=S>4 / 27$ some part of the liquid will be sliding down the wall because of the gravity.

If the surface tension effect is not neglected than $h(x, t)$ height of the thin liquid film can be modeled by:

$$
\frac{\partial h}{\partial t}+\left(3 h^{2}-2 h\right) \frac{\partial h}{\partial x}+\alpha \frac{\partial}{\partial x}\left(h^{3} \frac{\partial^{3} h}{\partial x^{3}}\right)=S(x),
$$

where the value of the parameter $\alpha$ controls how strong the surface tension is.

with initial condition given by

$$
h(x, 0)=h_{0} .
$$

For both numerical simulations, with weak and strong source, the initial data were taken $h(x, 0)=$ 0 . Time evolution for the weak source case is presented in Figure 16. At the initial time the liquid height just building up above the source localized at $[0,1$ segment, when it converges to a steady state on the interval $[0,1]$ and develops two, smoothed by the surface tension, shock waves moving with different velocities up the wall. The front wave is moving faster (and can not be modeled without taking into account surface tension (i.e by 4.1)). At the same time the following up wave, that is slower than the front one, admits a good approximation by the shock wave developed in PDE (4.1). 


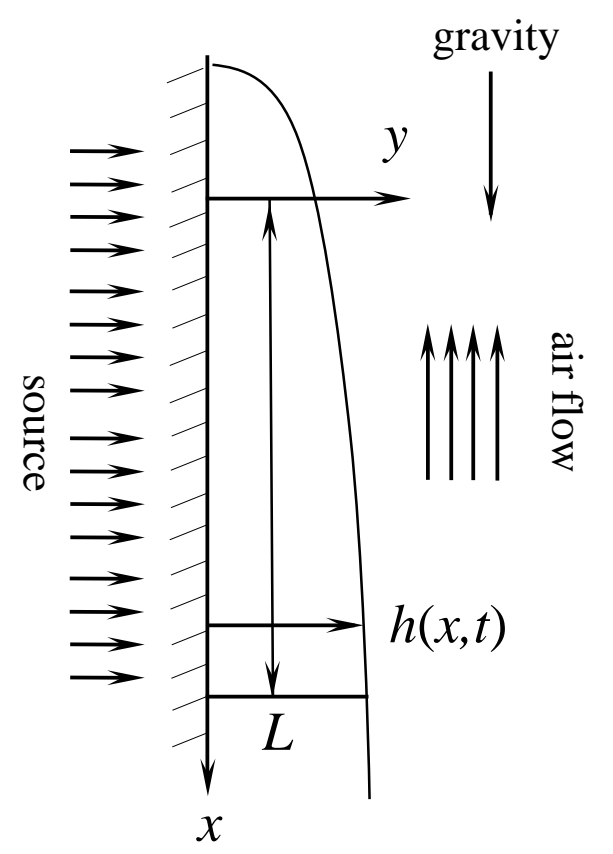

Figure 14: Thin film flowing under the influence of gravity, air flow and a source

Time evolution for the strong source is presented in Figure 17. Similar to the weak source case, at the initial time the liquid height just building up above the source localized at $[0,1$ segment and finally converges to a steady state on the interval $[0,1]$. The difference is that in the strong source case, shock waves are moving in two different directions, the second one continues to move up the wall but the front one turns around about the threshold $S>4 / 27$ and starts moving down the wall due to the gravity force. Shock wave that is moving down the wall can only have height that is greater than 1 . Increasing surface tension parameter value from $\alpha=0.001$ to $\alpha=0.01$ (see time evolution of the thin film height in Figure 18 will smooth shapes of the traveling waves.

\subsection{Characteristic equations}

The associated characteristic equations are

$$
\begin{aligned}
& \frac{d t(s)}{d s}=1 \\
& \frac{d x(s)}{d s}=3 z^{2}-2 z \\
& \frac{d z(s)}{d s}=S
\end{aligned}
$$

For $S$ is constant, we have,

$$
\begin{aligned}
& z(t)=S t \\
& x(t)=S^{2} t^{3}+\left(h_{0}-1\right) S t^{2}+\left(3 h_{0}^{2}-2 h_{0}\right) t+x_{0}
\end{aligned}
$$




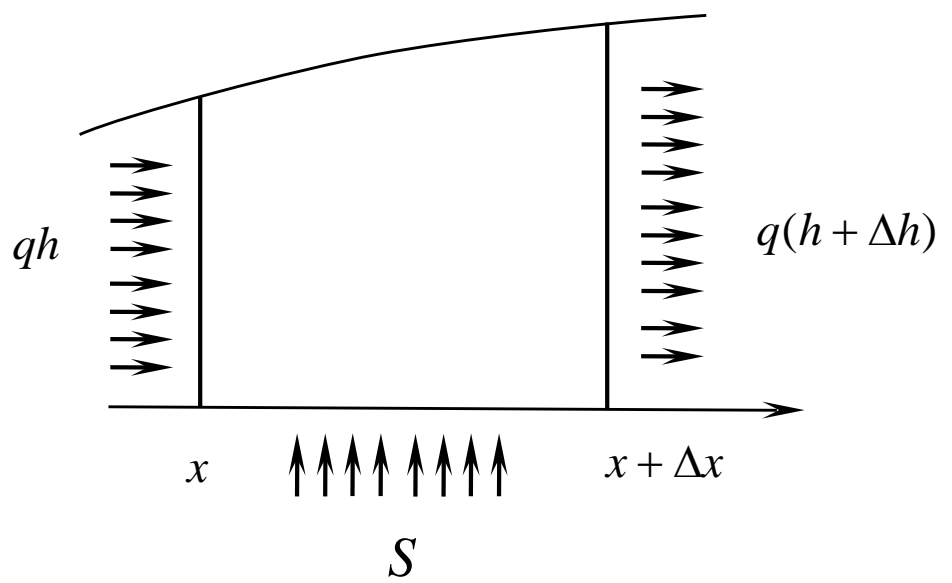

Figure 15: Small control volume along the length of the thin film

Under the case $h_{0}=0$, the characteristic line is reduce to

$$
x(t)=S^{2} t^{3}-S t^{2}+x_{0}
$$

where $x_{0}$ is a point on initial value curve.

\subsection{Steady states}

Setting $\frac{\partial h}{\partial t}=0$, we have,

$$
\left(3 h_{s}^{2}-2 h_{s}\right) \frac{\partial h_{s}}{\partial x}=S
$$

solve this we have,

$$
h_{s}^{3}-h_{s}^{2}=S x+C
$$

Now under assumption that

$$
\begin{aligned}
S & <<1 \\
h_{s}(1) & =0
\end{aligned}
$$

we have,

$$
h_{s}^{3}-h_{s}^{2}=S(x-1)
$$

On the other hand, if

$$
S>>1
$$

we have,

$$
h_{s}^{3}-h_{s}^{2}=S x
$$



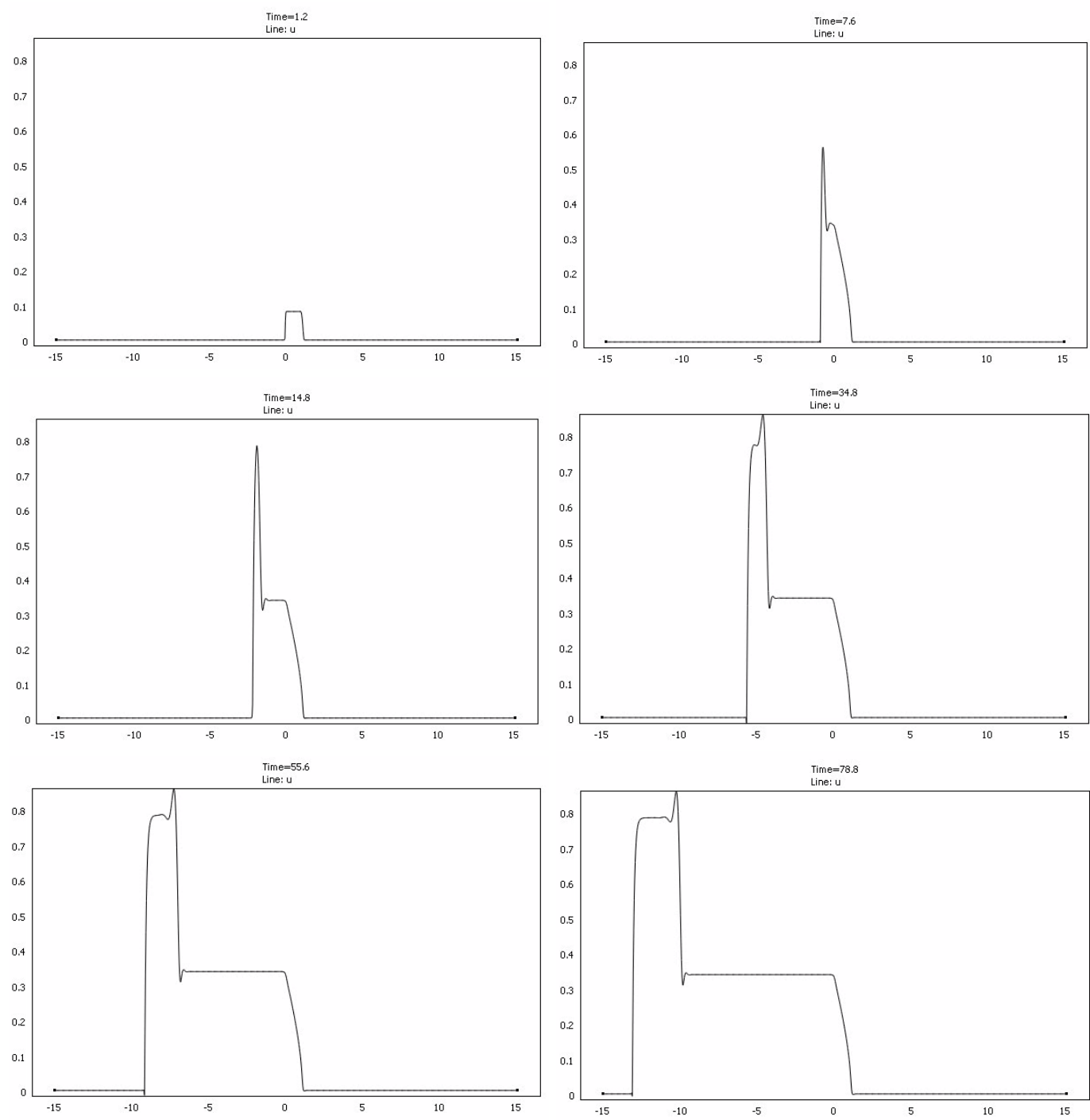

Figure 16: Thin film height time evolution for the weak source case $S=1 / 15<4 / 27$, periodic boundary conditions on the truncated interval $[-15,15]$ and with surface tension parameter $\alpha=0.001$.

\subsection{Connection between characteristic and steady states}

We observe inconsistency between the solution obtained by method of characteristic and steady states result. Here we try to find the locations or connections of our steady states solutions in our characteristic line result.

\subsubsection{Steady states under small source assumption}

First, we can see that the steady states solution under $\mathrm{S}$ small assumption is same as the characteristic line pass $x_{0}=1$ values. $h(x)=S t^{*}$, where $t^{*}$ is the time s.t. $x\left(t^{*}\right)=x$. This can be shown as,

$$
x\left(t^{*}\right)=S^{2}\left(t^{*}\right)^{3}-S\left(t^{*}\right)^{2}+1=x
$$




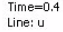

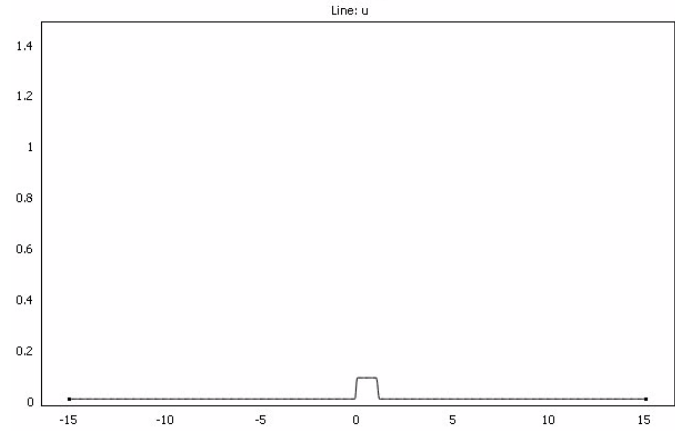

Time $=19.2$
Line: $U$
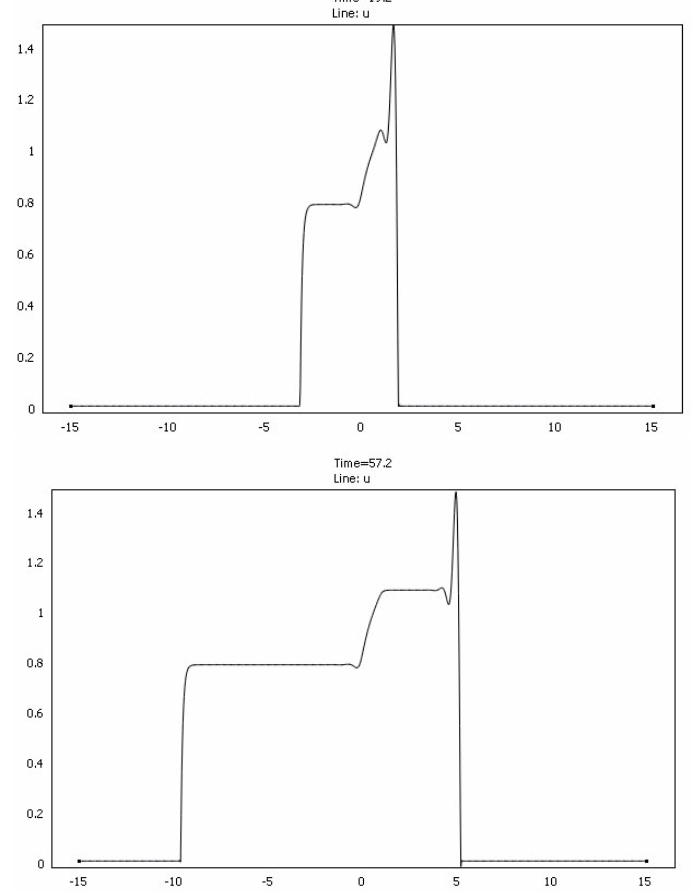

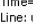

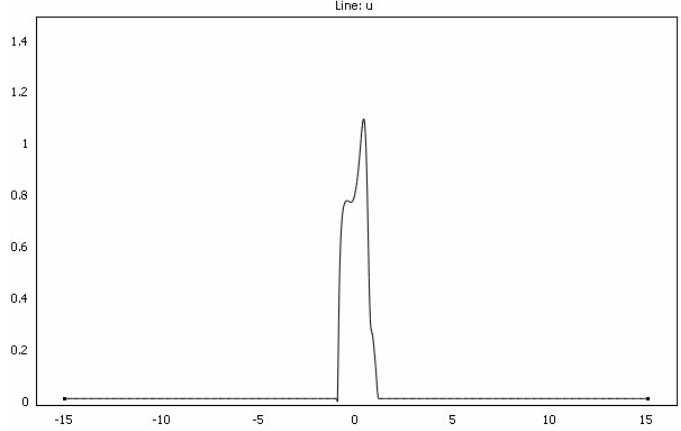

Time $=32.8$
Line: $u$

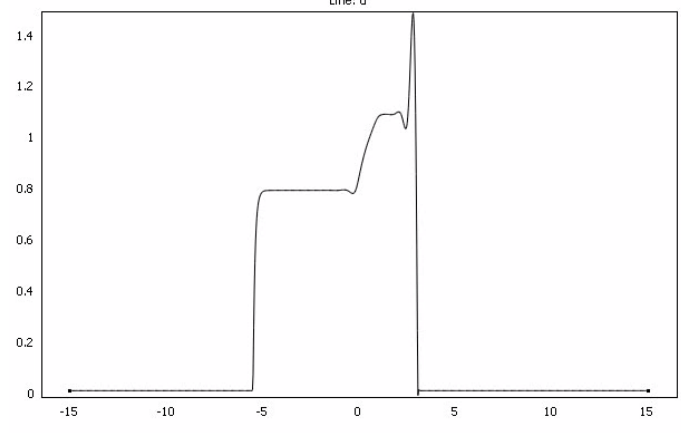

Time $=79.2$
Line: 4

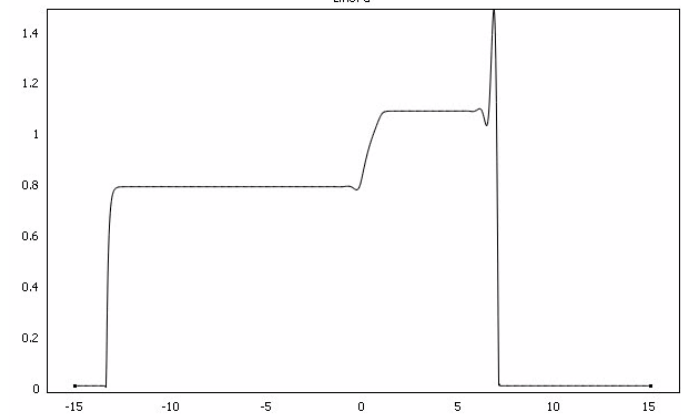

Figure 17: Thin film height time evolution for the strong source case $S=1 / 5>4 / 27$, periodic boundary conditions on the truncated interval $[-15,15]$ and with surface tension parameter $\alpha=0.001$.

Then multiply $\mathrm{S}$ on both side,

$$
S^{3}\left(t^{*}\right)^{3}-S^{2}\left(t^{*}\right)^{2}+S=S x
$$

Plug in $h(x)$, then

$$
h^{3}(x)-h^{2}(x)=S(x-1)
$$

The other way to see the steady state solution is the time of characteristics that pass $x$ at time $\frac{2}{3 S}$ stayed between $x=0$ and $x=1$ times source $S$.

The characteristic line pass $x$ at time $\frac{2}{3 S}$ is

$$
x(t)=S^{2} t^{3}-S t^{2}+x+\frac{4}{27 S}
$$

Let $t_{i}$ be the time this line intersection with $x=1$, we have,

$$
x\left(t_{i}\right)=S^{2} t_{i}^{3}-S t_{i}^{2}+x+\frac{4}{27 S}=1
$$



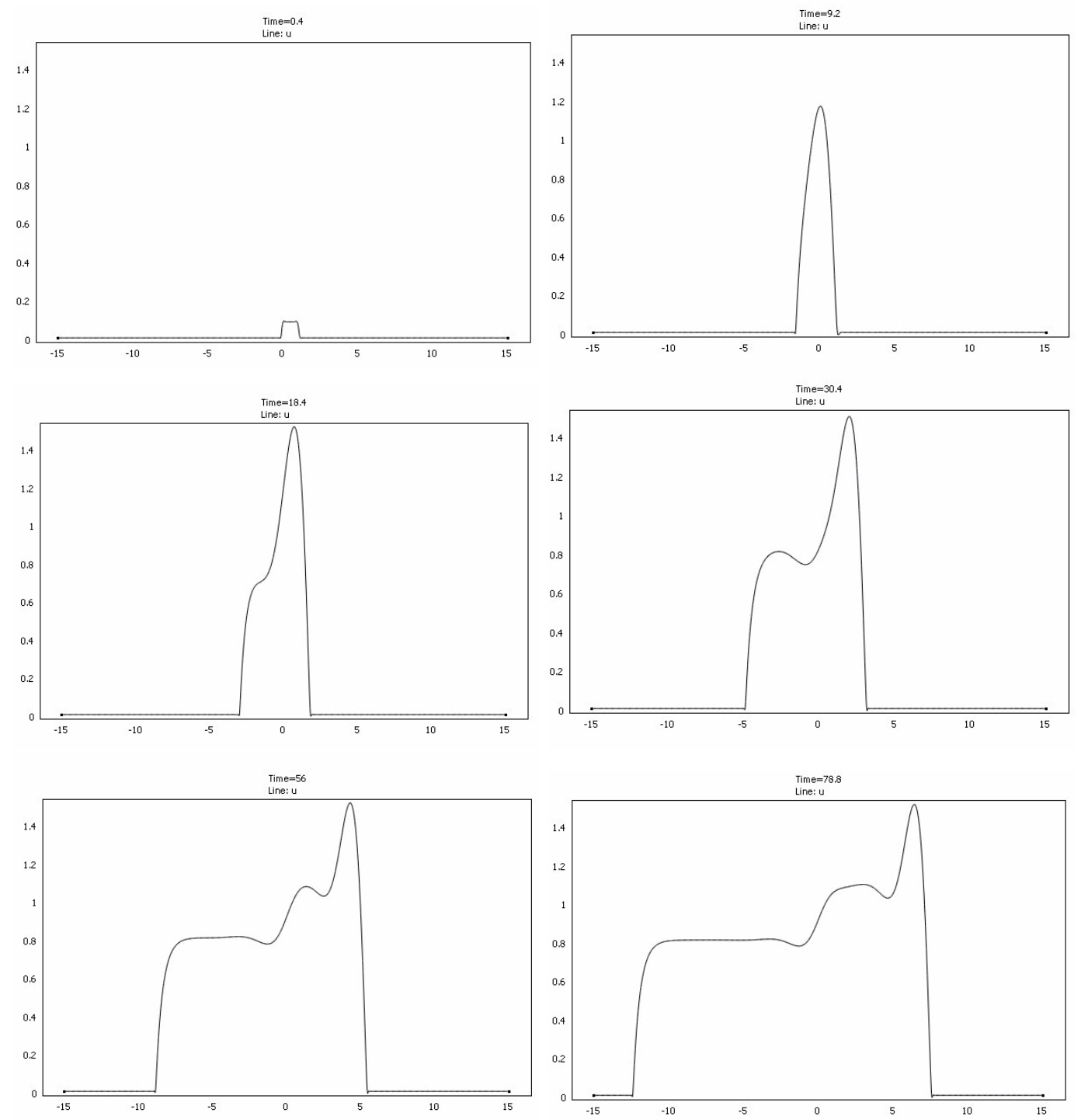

Figure 18: Thin film height time evolution for the strong source case $S=1 / 5>4 / 27$, periodic boundary conditions on the truncated interval $[-15,15]$ and with surface tension parameter $\alpha=0.01$.

and we have $h(x)=\left(\frac{2}{3 S}-t_{i}\right) * S$, plug in $S t_{i}=\frac{2}{3}-S$, and simplify, we have

$$
h^{3}(x)-h^{2}(x)=S(x-1)
$$

\section{Critical time estimate for surface clogging}

1. We consider the evolution of droplets of different radii on the surface. We assume that the droplet distribution is repetitive (similar on different distant parts of the surface). Then, let us consider blobs ${ }^{1}$ - rectangular parts of the surface with similar droplet distribution. The area of a blob is $A$.

2. The size (area) of a droplet increases thanks to the following two processes:

(a) Imbibing the liquid from the pores located in the absorbent from the pores inside the filter. This 
liquid is the result of chemical reaction of the sulfur gas flow through the pores

$$
2 \mathrm{SO}_{4}+2 \mathrm{H}_{2} \mathrm{O}+\mathrm{O}_{2}=2 \mathrm{H}_{2} \mathrm{SO}_{4} .
$$

(b) Coalescence.

\section{Mathematical model.}

Let $A(t)$ be the area of a rectangular blob at time $t$. The increase of the blob area happens thanks to the two above processes:

$$
d A=d A_{1}+d A_{2}
$$

where $d A_{1}$ is the increase thanks to the liquid imbibing from the pores

$d A_{2}$ is the increase thanks to coalescence (droplet merging).

Let $f, 0 \leq f \leq 1$ be the fraction of the blob's area covered by liquid. We assume that the input of liquid from the under-drop pores decreases to zero after the pore is covered by the droplet. Then

$$
d A_{1}=\alpha(1-f) A d t
$$

The increase of blobs thanks to coalescence, $d A_{2}$, is proportional to $4 d A_{1}$ (increase of the blob size towards four neighboring blobs) and is proportional to $8 A$ (eight blobs around the blob under consideration). Then,

$$
d A_{2}=4 \cdot 8 A d A_{1}=32 \alpha(1-f) A^{2} d t
$$

Finally,

$$
d A=d A_{1}+d A_{2}=\alpha(1-f)\left(A+32 A^{2}\right) d t .
$$

Thanks to the appearance of newly generated droplets, it is naturally to assume

$$
\frac{d f}{d t}=\beta(1-f)
$$

Then

$$
f=1-e^{-\beta t}
$$

and we obtain the governing equation

$$
\frac{d A}{d t}=\alpha e^{-\beta t} A(1+32 A)
$$

Its solution is

$$
A(t)=\frac{A_{0}}{\left(1+32 A_{0}\right) \exp \left(-\frac{\alpha}{\beta}(1-\exp (-\beta t))\right)-32 A_{0}}
$$

This solution becomes infinite (i.e., the surface is completely covered by film) at the critical time

$$
t_{c r}=\frac{1}{\beta} \ln \frac{1}{1-\frac{\beta}{\alpha} \ln \left(1+\frac{1}{32 A_{0}}\right)}
$$

which is finite if

$$
\frac{\beta}{\alpha} \ln \left(1+\frac{1}{32 A_{0}}\right)<1
$$

or

$$
A_{0}>\frac{1}{32\left(e^{\alpha / \beta}-1\right)} \text {. }
$$




\section{Estimation of the parameters}

Returning back, we actually solve equation

$$
\frac{d A}{d t}=b A+c A^{2}
$$

with the difficulties to estimate initial condition $A_{0}$. To estimate $b, c, A_{0}$, several observations are required in order to evaluate the fraction of the area covered by the droplets (the value of $f$ ).

\section{References}

AfKhami, S., Zaleski, S. \& Bussman, M. 2009 A mesh-dependent model for applying dynamic contact angles to VOF simulations. J. Comput. Phys. 228, 5370-5389.

Agbaglah, G., Delaux, S., Fuster, D., Hoepffner, J., Josserand, C., Popinet, S., Ray, P., Scardovelli, R. \& Zaleski, S. 2011 Parallel simulation of multiphase flows using octree adaptivity and the volume-of-fluid method. Comptes Rendus Mecanique 339 (2), 194-207.

Cimpeanu, R. \& PAPAgeorgiou, D.T. 2015 Electrostatically induced mixing in confined stratified multi-fluid systems. Int. J. Multiphase Flow 75, 194-204.

Cimpeanu, R. \& Papageorgiou, D.T. 2018 Three-dimensional high speed drop impact onto solid surfaces at arbitrary angles. International Journal of Multiphase Flow 107, 192 - 207.

Cimpeanu, R., Papageorgiou, D.T. \& Petropoulos, P.G. 2014 On the control and suppression of the Rayleigh-Taylor instability using electric fields. Physics of Fluids (1994-present) 26 (2), 022105.

DEBAR, R. 1974 Fundamentals of the KRAKEN code. Lawrence Livermore Laboratory, UCIR-760 .

López-Herrera, J.M., Popinet, S. \& HerradA, M.A. 2011 A charge-conservative approach for simulating electrohydrodynamic two-phase flows using volume-of-fluid. J. Comput. Phys. 230, 1939-1955.

Popinet, S. 2003 Gerris: A tree-based adaptive solver for the incompressible Euler equations in complex geometries. J. Comput. Phys. 190, 572.

Popinet, S. 2009 An accurate adaptive solver for surface-tension-driven interfacial flows. J. Comput. Phys. 228, 5838.

Tryggvason, G., Scardovelli, R. \& Zaleski, S. 2011 Direct numerical simulations of gasliquid multiphase flows. Cambridge University Press. 